\title{
Poster Previews for Conference 9907: Optical and Infrared Interferometry and Imaging $\mathrm{V}$
}

, "Poster Previews for Conference 9907: Optical and Infrared Interferometry and Imaging V," Proc. SPIE 10015, Poster Previews: SPIE Astronomical Telescopes and Instrumentation 2016, 1001505 (16 June 2016); doi: $10.1117 / 12.2248393$

SDIE Event: SPIE Astronomical Telescopes + Instrumentation, 2016, Edinburgh, United Kingdom 


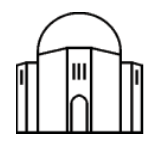

STEWARD OBSERVATORY

\section{Fundamental gain in high contrast imaging with the}

F. Patru, S. Esposito, A. Puglisi, A. Riccardi, E. Pinna, C. Arcidiacono, J. Hill, P. Hinz Osservatorio Astrofisico di Arcetri, Firenze, Italia \& LBT Observatory, Tucson, United States

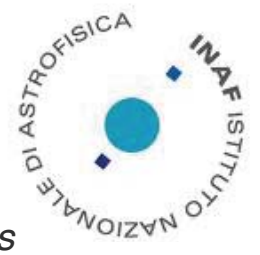

\section{ABSTRACT}

Numerical simulations for the LBTI have shown a fundamental gain in contrast when using two $8 \mathrm{~m}$ adaptive optics telescopes instead of one. The LBTI provides an "in-between" PSF, combining both diffractive and interferometric properties (Fig. 1). The maximum intensity in the PSF is increased by a factor of four, accounting for the double flux and the fringe intensification. The imaging performance of the LBTI is limited by the AO-correction level, as for a single LBT aperture (Fig. 3,4). The PSFs are computed by using 2 time series of residual wavefronts with an RMS of $\sim 100 \mathrm{~nm}$ yielding an AO Strehl of $50 \%$ in $\mathrm{R}$ band (visible). The contrast gain map is defined as the normalised ratio of the PSF of a single LBT over the PSF of the LBTI. The contrast gain averaged across the field is improved by a factor 2 in contrast by using the long exposures and by a factor of 10 in contrast by using the short exposures (Fig. 5,6). Indeed, contrary to the long exposure where the fringes are blurred, a snapshot retains the fringes formed in a halo of speckles (Fig. 3), enabling speckle imaging. The speckle halo contains not only high angular resolution information but also high contrast imaging information. Thus, there is some gain in grouping some short exposures with high gain. A gain zone is produced in the valleys of the PSF formed by the dark Airy rings and/or the dark fringes. A huge contrast gain $(G \sim 100$ to 1000) in narrow zones (few mas) can be achieved when both a dark fringe and a dark ring overlap. The Earth rotation allows to exploit various area in the contrast gain map in which a planet passes through several gain zones. This makes the LBTI well suitable for the Angular Differential Imaging (ADI) technique. A rotation of $15^{\circ}$ is sufficient to pass through at least one contrast gain zone (Fig. 7). A planet is located in a white or in a dark ring, depending on its radial distance. A planet is alternatively located in a white fringe $(G \sim 1)$ or in a dark fringe ( $G \sim 10$ to 100$)$ as a function of the parallactic angle (Fig. 8,9).

\section{Diffraction-limited PSF}

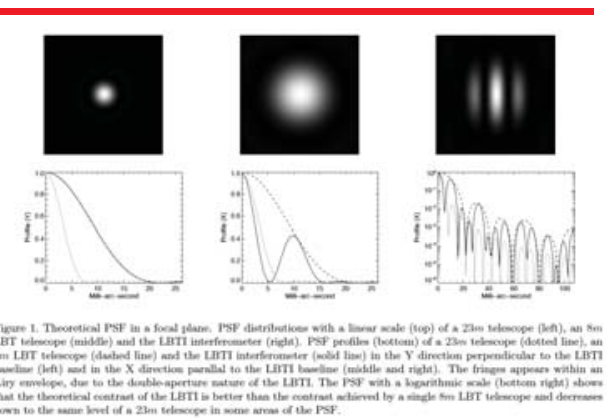

\section{Contrast gain map $=4 \times$ PSF(LBT) / PSF(LBTI)}

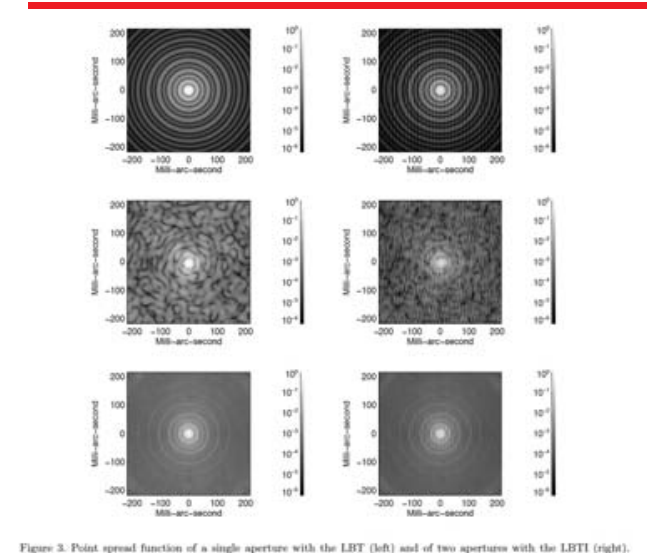

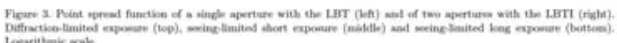
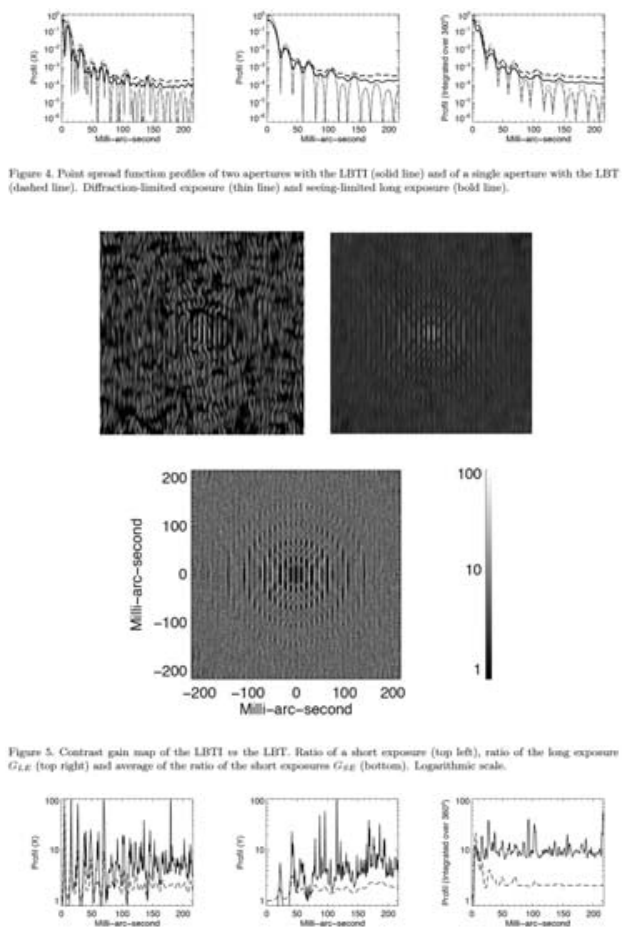

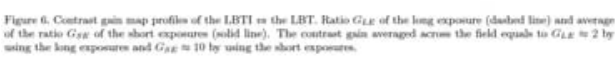

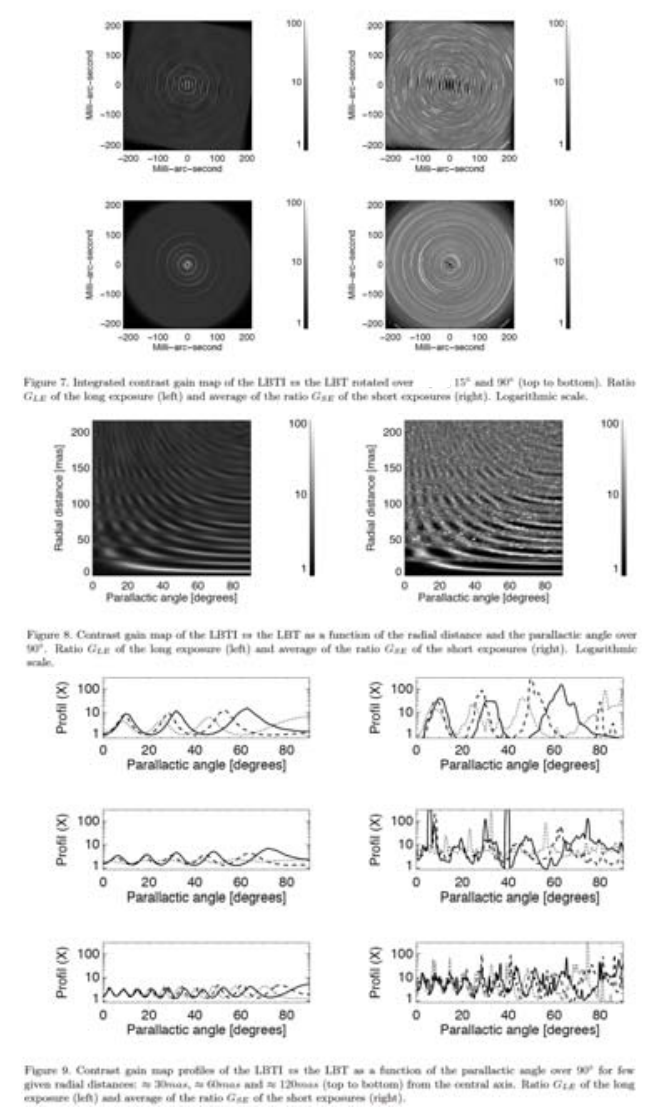

\section{CONCLUSIONS}

Compared to a standalone $8 \mathrm{~m}$ LBT, the $23 \mathrm{~m}$ LBTI can provide not only a gain in angular resolution (by a factor of 3 to 10 in the infrared vs visible) and a gain in sensitivity (by a factor of 4), but also a gain in contrast (by a factor of 10 to 1000), assuming a high AO Strehl and a cophasing mode. A new ADI-like mode should be developed for high contrast imaging in the visible wavelengths in the framework of the LIVE project. Indeed, the visible PSF contains thinner structures than the infrared PSF, providing numerous dark fringes and dark rings where planets can be detected at low level.
Further information by e-mail : fabienpatru@gmail.com esposito@arcetri.astro.it

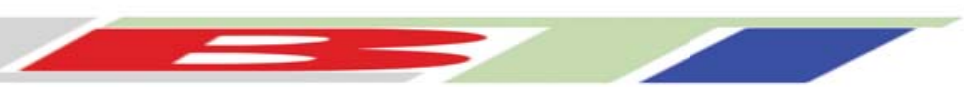

SPIE.

Poster Previews: SPIE Astronomical Telescopes and Instrumentation 2016

Proc. of SPIE Vol. 10015, 1001505 - (c) 2016 SPIE · CCC code:

0277-786X/16/\$18 - doi: $10.1117 / 12.2248393$ 


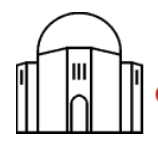

STEWARD

\title{
Sensitivity to piston \& adaptive optics errors with the
}

\author{
F. Patru, S. Esposito, A. Puglisi, A. Riccardi, E. Pinna, C. Arcidiacono, J. Hill, P. Hinz
} Osservatorio Astrofisico di Arcetri, Firenze, Italia \& LBT Observatory, Tucson, United States

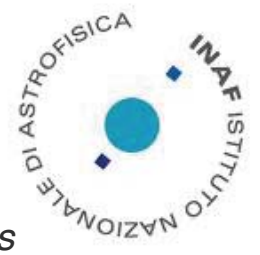

\section{ABSTRACT}

On-sky adaptive optics wavefront screens have been used and random optical path fluctuations - differential pistons - have been included in numerical simulations for the LBTI. The interferometric Strehl which can be achieved by the LBTI using two FLAO is close to the AO Strehl provided by a FLAO standalone, as long as other sources of aberrations can be mitigated (Fig. 1). The imaging performance of the LBTI is thus fundamentally limited by the AOcorrection level. The tip-tilts induced by both $\mathrm{AO}$ systems onto each sub-aperture produce differential tip-tilts of the sub-beams and differential shifts of the sub-images (Fig. 2, 4, 5), so that the diffraction envelopes do not overlap properly and the beams do not fully interfere coherently. However, for piston fluctuations, the fringes move back and forth within the Airy disk (Fig. 3, 4,5), reducing the fringe contrast for a long exposure, but not for a short one. A snapshot retains high angular resolution information by freezing the fringes. Few criteria have been evaluated as a merit function (Fig. 6, 7) to characterise the Point Spread Function (PSF) and the Optical Transfer Function (OTF). The R23 quantity is more sensitive than the interferometric Strehl to the contrast attenuation induced by the shift of the fringes in a long exposure (image blurring). The visibility should not be used as a merit function, but as a criterium for science applications. The contrast in the PSF enables to highlight two regimes depending on the location in the diffractive pattern, either in a sidelobe (low contrast) or in a valley (high contrast). The FWHM of the central peak of the MTF may be used to sense the low order aberrations from each $\mathrm{AO}$ systems. For instance, large tip-tilts produce fringes in the central peak affecting the FWHM in real-time.

\section{FLAO wavefronts}

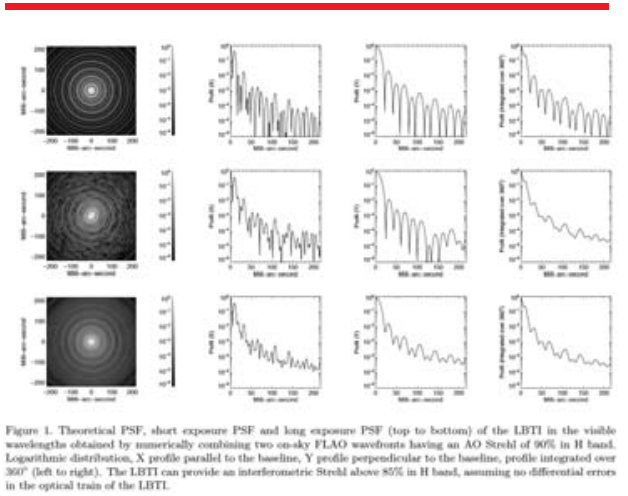

\section{Pistons \& AO errors}

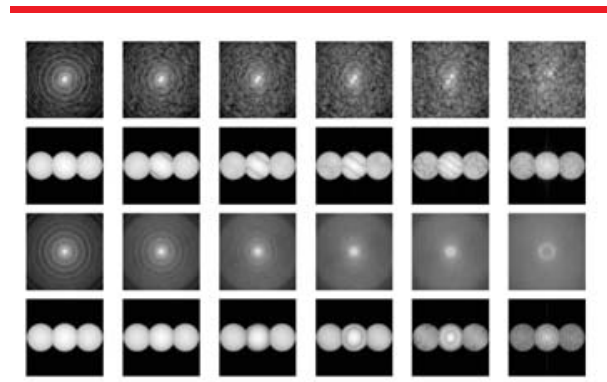

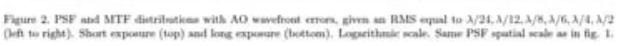
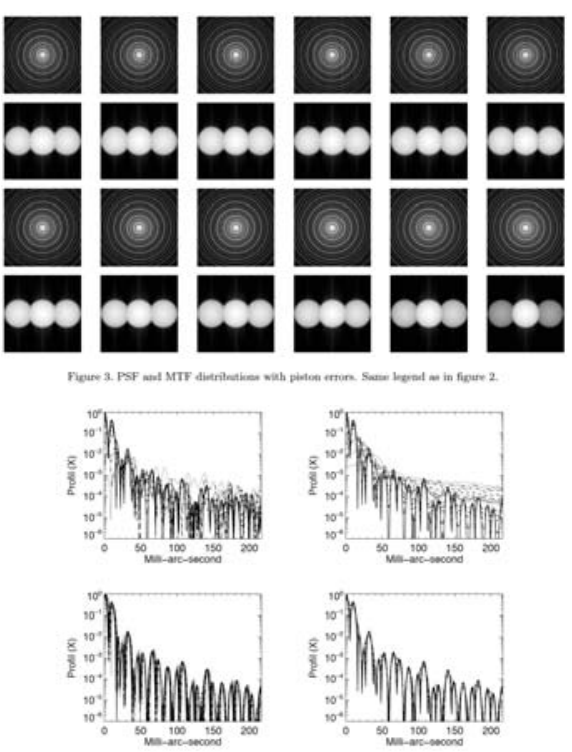

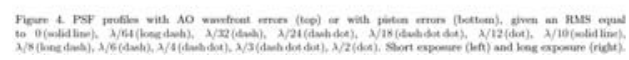
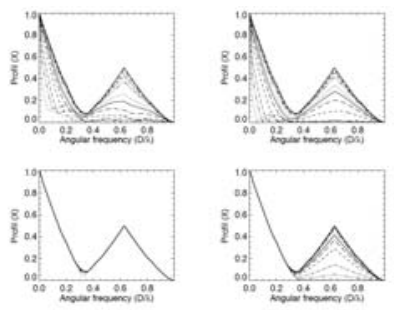

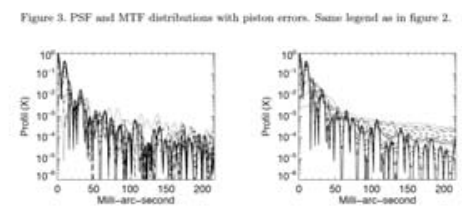

\section{PSF \& MTF criteria}

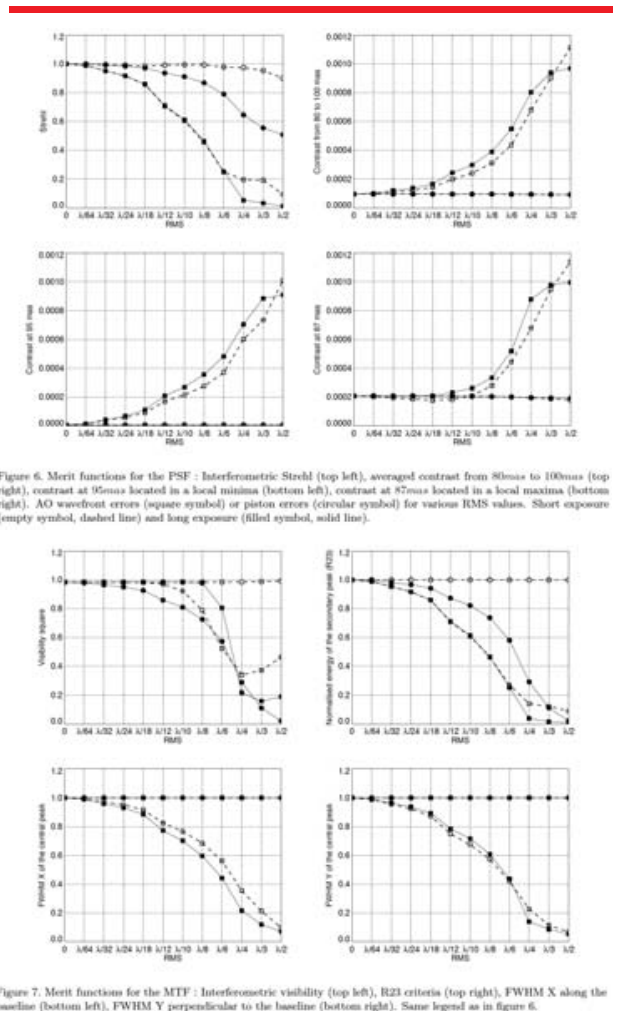

\section{CONCLUSIONS}

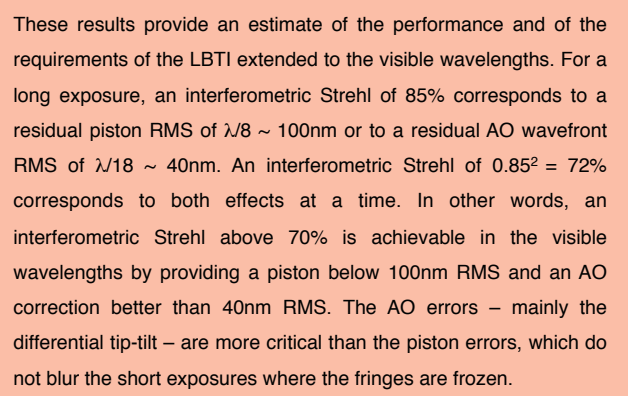

Further information by e-mail :

fabienpatru@gmail.com

esposito@arcetri.astro.it
SPIE. 


\title{
GRAVITY: the new metrology concept
}

\author{
N. Blinda, Y. Kok, F. Eisenhauer, S. Gillessen, M. Lippa \& the GRAVITY consortium \\ Max-Planck-Institut für Extraterrestrische Physik (Germany)
}

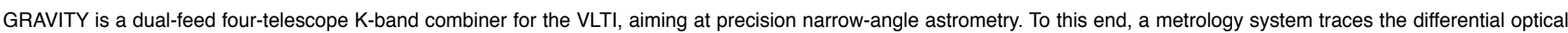

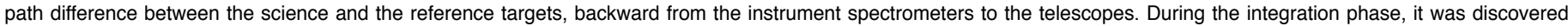

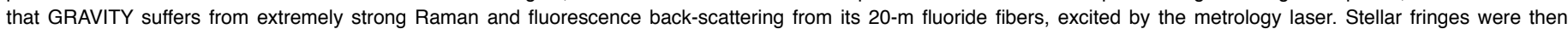
contaminated on the spectrometers detectors by a strong incoherent light background, dramatically reducing the instrument sensitivity by more than 10 stellar magnitudes!

We present here the concept of the new metrology system that mitigates this back-scattering effect to a level such that GRAVITY can operate with optimal performance. More details on the implementation of this system can be found in Poster 9907-73.

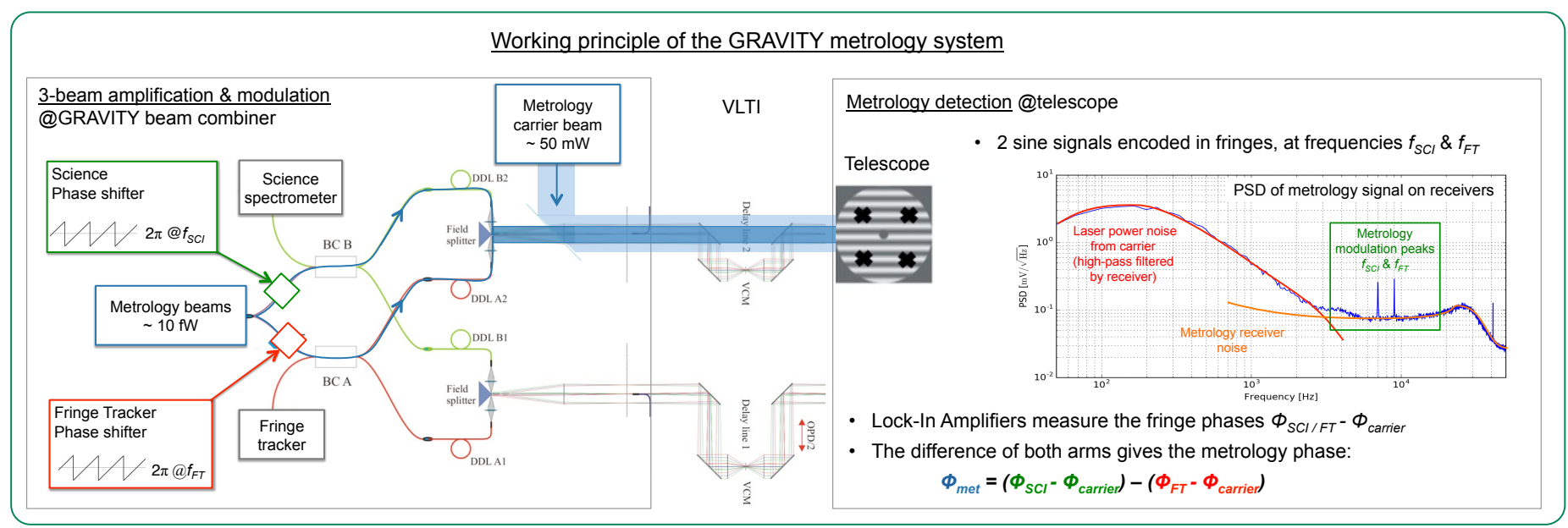

\section{3-BEAM OPTICAL AMPLIFICATION}

- Interference of 2 metrology beams:

$$
F=I_{1}+I_{2}+\sqrt{I_{1} I_{2}} \cos \left(\phi_{12}\right)
$$

- Metrology phase SNR $\propto \sqrt{I_{1} I_{2}}$

$\rightarrow$ The addition of a $3^{\text {rd }}$ high power carrier beam $(>50 \mathrm{~mW})$ allows decreasing the laser power in fibers down to fW level at constant SNR.

- Back-scattering and non-common path effects (e.g. differential fiber heating) reduced in proportion, by a factor $100-1000$, hence are negligible now.

\section{SYNCHRONOUS DETECTION}

- Phase-shifters sawtooth modulation of amplitude $2 \pi$ and frequencies $f_{\mathrm{SCl}} \& f_{\mathrm{FT}}$ between 10 and $20 \mathrm{kHz}$

$\rightarrow$ Telescope fringe signal $F \sim$ pure sine harmonic $1 f$

- Lock-In Amplifiers (LIA) demodulate the sine fringe signal and measure phase $\Phi_{S C I / F T}-\Phi_{\text {carrier }}$

- $\mathrm{LIA}=$ narrowband filter $(\Delta f<100 \mathrm{~Hz})$ at frequency $f$

$>$ disentangle both metrology signals

$>$ Filter receivers white noise, improving metrology sensitivity by a factor "few" wrt ABCD

$>$ filter out the three-beam laser power fluctuations, vibrations, etc

$>$ very flexible with the free choice of modulation frequencies wrt noises

\section{RESULTS}

This new metrology system mitigates the backscattering issue, allowing optimal operations of GRAVITY. It also reduced NCP effects to a negligible level and proved to be more sensitive and flexible than a standard $A B C D$ modulation.

The metrology system of GRAVITY has been upgraded in consequence with:

- 4 additional carrier laser beams of $\sim 50 \mathrm{~mW}$ each;

- 40 Lock-In Amplifiers measuring the phase from the two metrology arms;

- All 40 metrology receivers were also modified to deal with too high laser power fluctuations.

See poster $\mathbf{9 9 0 7 - 7 3}$ for more details on the implementation.

a The author moved to the Observatory of Geneva since this work was done. Current contact email: nicolas.blind@unige.ch 
A new dual-channel speckle instrument for Gemini and WIYN, Paper Number: 9907-100

Nic J Scott ${ }^{1}$, Steve Howell ${ }^{1}$, Elliott Horch ${ }^{2}$

NASA Ames Research Center

\section{Introduction}

Speckle imaging allows telescopes to achieve diffraction limited imaging performance. The technique requires cameras capable of reading out frames at a very fast rate, effectively 'freezing out' atmospheric seeing. The resulting speckles can be correlated and images reconstructed that are at the diffraction limit of the telescope. These new instruments are based on the successful performance and design of the Differential Speckle Survey Instrument (DSSI) $[2,1]$

The instruments are being built for the Gemini-N and WIYN telescopes and will be made available to the community via the peer review proposal process. We envision their primary use to be validation and characterization of exoplanet targets from the NASA K2 and TESS missions and RV discovered exoplanets. Such targets will provide excellent follow-up candidates for both the WIYN and Gemini telescopes [3]. Examples of DSSI data are shown in the figures below. We expect similar data quality in speckle imaging mode with the new instruments.

Additionally, both cameras will have a wide-field mode and standard SDSS filters. They will be highly versatile instruments and it is that likely many other science programs will request time on the cameras. The limiting magnitude for speckle observations, will remain around 13-14th at WIYN and 16-17th at Gemini, while wide-field, normal CCD imaging operation should be able to go to much fainter, providing usual CCD imaging and photometric capabilities. The instruments will also have high utility as scoring cameras for telescope engineering purposes, or other applications where high time resolution is needed. Instrument support will be provided, including a software pipeline that takes raw speckle data to fully reconstructed images.

\section{Gemini North}

$\begin{array}{ll}\text { Telescope } \mathrm{f} / \# & 16.0 \\ \text { Plate scale } & 1.592^{\prime \prime} / \mathrm{mm}\end{array}$

GemSpeck - Speckle mode

$\begin{array}{ll}\mathrm{L} 1 & 35 \mathrm{~mm} \\ \mathrm{~L} 2 & 75 \mathrm{~mm}\end{array}$

Detector Image Plane

$\begin{array}{ll}\text { Magnification } & 2.14 \mathrm{x} \\ \text { Pixel Scale } & 0.0096^{\prime \prime} / \mathrm{pxl} \\ \text { Unvignetted Circle Dia } & 6.7^{\prime \prime}\end{array}$

Unvignetted Circle Dia $6.7^{\prime \prime}$

Detector FoV $\quad 9.9 \times 9.9^{\prime \prime}$

GemSpeck - Wide-Field mode Focal Lengths

$\begin{array}{lr}\mathrm{L} 1 & 75 \mathrm{~mm} \\ \mathrm{~L} 2 & -100 \mathrm{~mm} \\ \mathrm{~L} 3 & 50 \mathrm{~mm}\end{array}$

Detector Image Plane

$\begin{array}{ll}\text { Magnification } & 0.285 \mathrm{x} \\ \text { Pixel Scale } & 0.0725 " / \mathrm{pxl} \\ \text { Unvignetted Circle Dia } & 60^{\prime \prime} \\ \text { Detector FoV } & 74 \times 74^{\prime \prime}\end{array}$

Detector FoV Focal Lengths

\section{WIYN}

$$
\begin{aligned}
& \text { Telescope } \mathrm{f} / \# \\
& \text { Plate scale }
\end{aligned}
$$$$
6.289
$$

$9.374^{\prime \prime} / \mathrm{mm}$

WIYNSPKL - Speckle mode Focal Lengths

$\begin{array}{lr}\mathrm{L} 1 & 30 \mathrm{~mm} \\ \mathrm{~L} 2 & 200 \mathrm{~mm}\end{array}$

Detector Image Plane

$\begin{array}{ll}\text { Magnification } & 6.67 \mathrm{x} \\ \text { Pixel Scale } & 0.0182^{\prime \prime} / \mathrm{pxl} \\ \text { Unvignetted Circle Dia } & 22^{\prime \prime}\end{array}$

Unvignetted Circle Dia 22

Detector FoV $19 \times 19^{\prime \prime}$

WIYNSPKL - Wide-field mode Focal Lengths

$\begin{array}{ll}\mathrm{L} 1 & 100 \mathrm{~mm} \\ \mathrm{~L} 2 & 150 \mathrm{~mm}\end{array}$

Detector Image Plane

Magnification $\quad 1.5 x$

Pixel Scale $\quad 0.0813^{\prime \prime} / \mathrm{pxl}$

Unvignetted Circle Dia $56^{\prime \prime}$

Detector FoV $83 \times 83^{\prime \prime}$

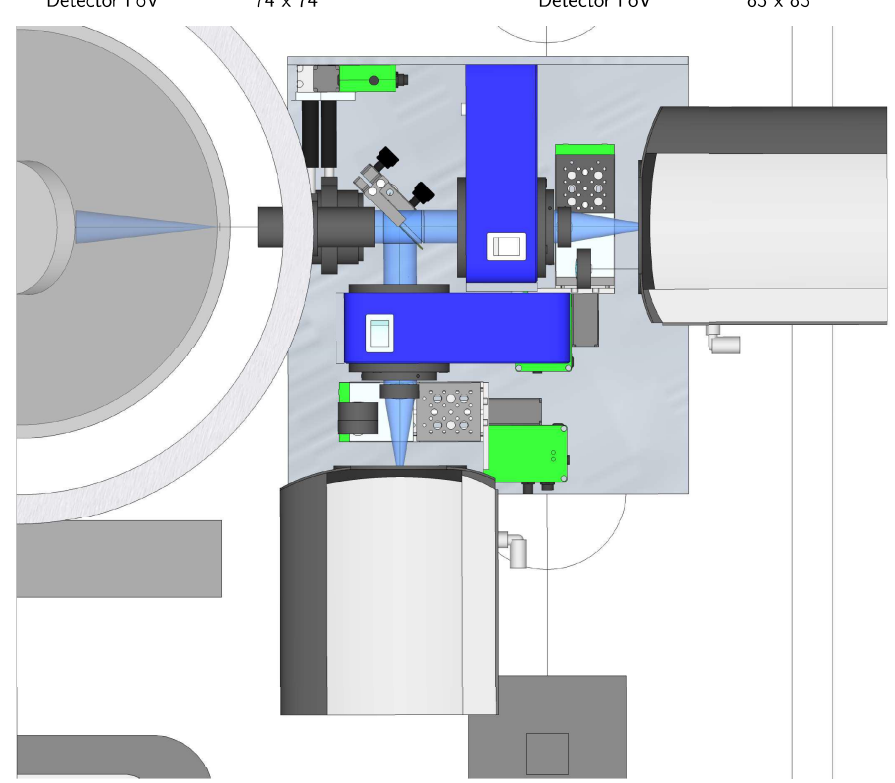

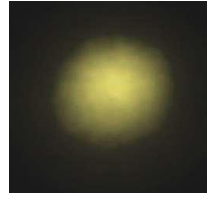

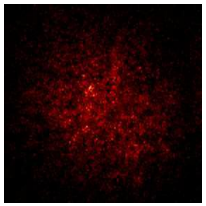

Integrated

Speckles

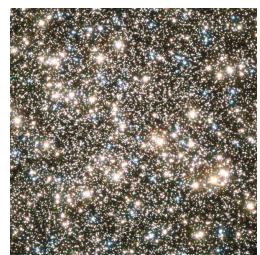

60" of M13 from HST archive. This is a a higher resolution image than we expect from the ground, but at a scale comparable to what will be available in WF mode.
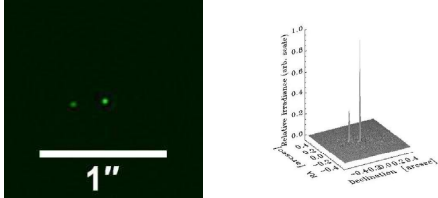

Reconstructed speckle image

Reconstructed speckle image

\section{Dichroic Edge $\quad 685 \mathrm{~nm}$}

This allows the blue $(447 \mathrm{~nm})$ and green $(562 \mathrm{~nm})$ filters in the reflective channel and the red $(692 \mathrm{~nm})$ and infrared $(880 \mathrm{~nm})$ filters in the transmissive channel.

$\begin{array}{cccc}\text { Filter Wheel A } & \text { central } \lambda \text {, bandwidth } & \text { Filter Wheel B } & \text { central } \lambda \text {, bandwidth } \\ \text { SDSS } / \mathrm{g} & 480 \mathrm{~nm}, 140 \mathrm{~nm} & \text { SDSS } / i & 770 \mathrm{~nm}, 150 \mathrm{~nm} \\ \text { SDSS } / \mathrm{r} & 625 \mathrm{~nm}, 140 \mathrm{~nm} & \text { SDSS } / \mathrm{z} & 910 \mathrm{~nm}, 120 \mathrm{~nm} \\ \text { g-narrow } & 466 \mathrm{~nm}, 40 \mathrm{~nm} & \text { i-narrow } & 692 \mathrm{~nm}, 40 \mathrm{~nm} \\ \text { r-narrow } & 562 \mathrm{~nm}, 40 \mathrm{~nm} & \text { z-narrow } & 832 \mathrm{~nm}, 40 \mathrm{~nm}\end{array}$

\section{Detectors}

The instrument will use two identical Andor iXon Ultra 888 EMCCD cameras.

- $1024 \times 1024$ with $13 \mu \mathrm{m}$ square pixels

- Capable of $26 \mathrm{fps}$ reading out the full chip, higher for subarray readout (speckle mode)

- EX coating, > 80\% quantum efficiency from 420 to $780 \mathrm{~nm},>90 \%$ QE between 550 and $720 \mathrm{~nm}$.

- Thermoelectrically cooled, require no consumables

- Data is transferred to the control computer via USB3, no internal cards

- Control computer can be quite small with heat dissipation being minimal.

Maximum Resolution

$\begin{array}{lll}\text { Gemini } & 0.015 \text { " FWHM @ } 500 \mathrm{~nm} & 0.025 \text { " FWHM @ } 800 \mathrm{~nm} \\ \text { WIYN } & 0.036 " \text { FWHM @ } 500 \mathrm{~nm} & 0.058 \text { " FWHM @ } 800 \mathrm{~nm}\end{array}$

\section{Discussion}

Possible Exoplanet Applications

- Simultaneous two color transit photometry yields instant verification (same depth in both channels).

- Standard imaging provides host star photometry

- Speckle imaging assesses binarity and yielding correct exoplanet radius

\section{Future Expansion}

The filter wheels each have two remaining empty slots, we are currently exploring possible uses for these including the addition of:

- Transmission Grating - Grism

- Aperture Mask

Possible science application include: exoplanet transit spectroscopy, exoplanet atmosphere detection, transient object classification and characterization.

An aperture mask would allow spatial resolution beyond the diffraction limit! Achieving true interferometric resolution (2.44x the diffraction limit). This could be especially interesting if used on next-generation ELTs.

\section{Acknowledgments}

We acknowledge the collaborations

\section{Notes}

NASA Ames Research Center, n.j. scottenasa gov, steve. b. howell@ nasa gou

Southern Connecticut State University, horche2 $\varrho_{\text {southernct.ed }}$

\section{References}

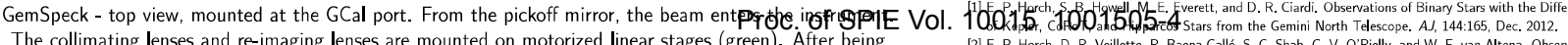

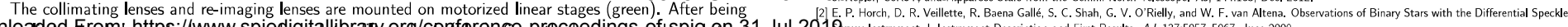

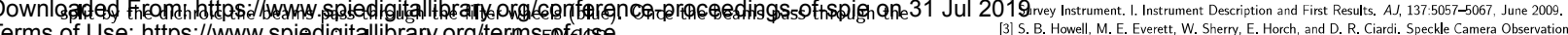




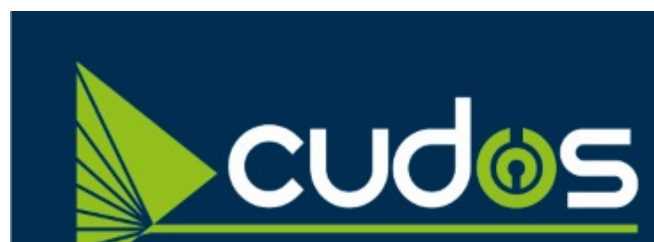

An ARC Centre of Excellence

Paper Number: 9907-109

\section{Chalcogenide Glass Planar MIR couplers for future chip based Bracewell Interferometers}

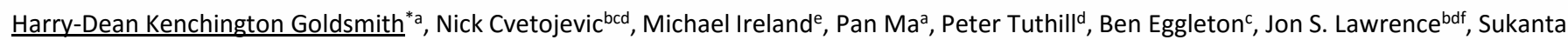
Debbarma $^{a}$, Barry Luther-Davies ${ }^{a}$, Stephen J. Madden ${ }^{\text {a }}$

${ }^{a}$ Centre for Ultrahigh bandwidth Devices for Optical Systems (CUDOS), Laser Physics Centre, Research School of Physics and Engineering, Australian National University, ACT, 2601, Australia; bThe Australian Astronomical Observatory (AAO), Level 1, 105 Delhi Rd, North Ryde, NSW 1670, Australia;

'Centre for Ultrahigh bandwidth Devices for Optical Systems (CUDOS), School of Physics, University of Sydney, NSW 2006, Australia

'Sydney Institute for Astronomy (SIfA), School of Physics, University of Sydney, NSW 2006, Australia;

eResearch School of Astronomy \& Astrophysics, Australian National University, ACT 2611, Australia;

${ }^{f} \mathrm{MQ}$ Photonics Research Centre, Department of Physics and Engineering, Macquarie University, NSW 2109, Australia.

The fundamental goal of exoplanet research is to find Earth-like bodies. Already there are discoveries of rocky planets orbiting in their respective habitable zones. The inevitable future of exoplanet exploration is the characterisation of the atmosphere of these planets. To achieve this exoplanets must be imaged, using interferometry, with high angular resolution and extreme dynamic range. Here we present a photonic approach that enables such astronomy by using a Bracewell inspired nulling interferometer. The idea is to use a single photonic chip, made of layers of Chalcogenide glass, to null the star light enabling characterisation of the exoplanet light.
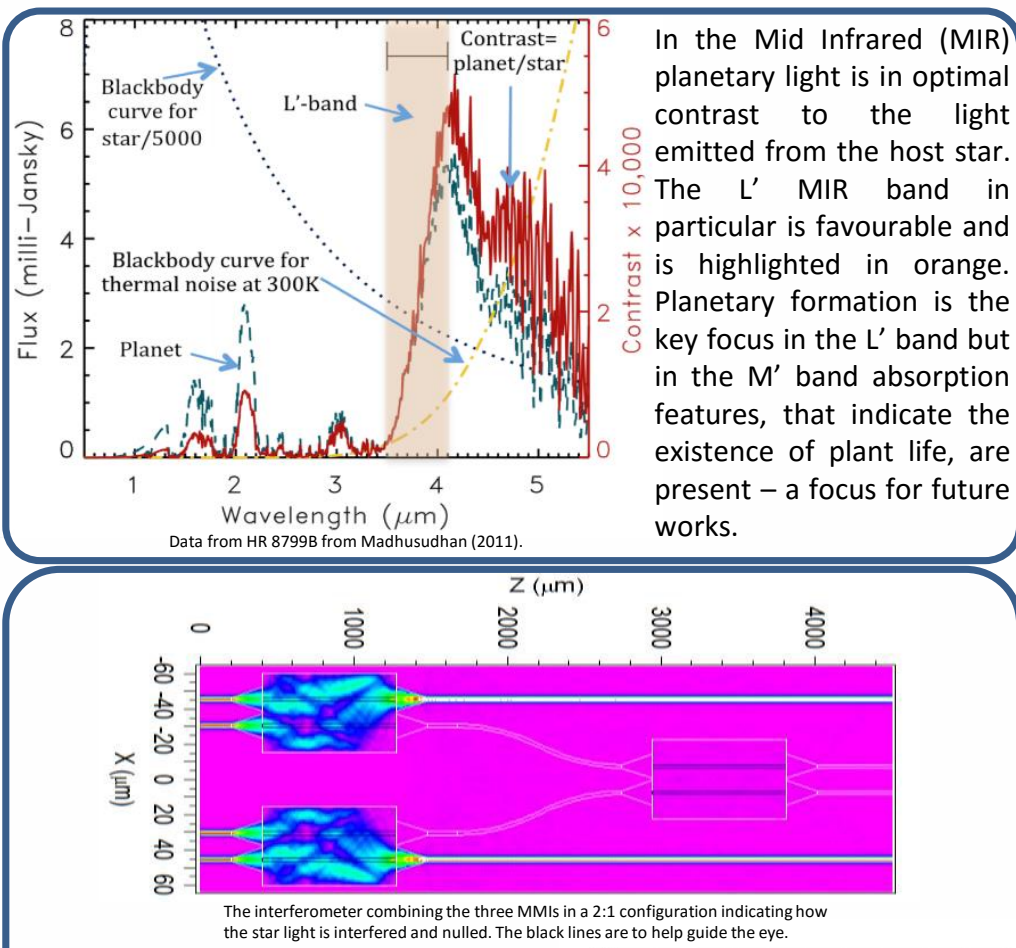

The interferometer allows a planet to be found orbiting a star when otherwise the star light would be too bright. The combination of three MMls in the figure above indicates the path of the star light allowing the third MMI to identify light not from the star, such as a planet or merely dust.

The Extinction Ratio is a measure of how effectively the star has been nulled. The right side picture provides an indication of the effectiveness of the device purely based on the simulations of the individual MMIs.

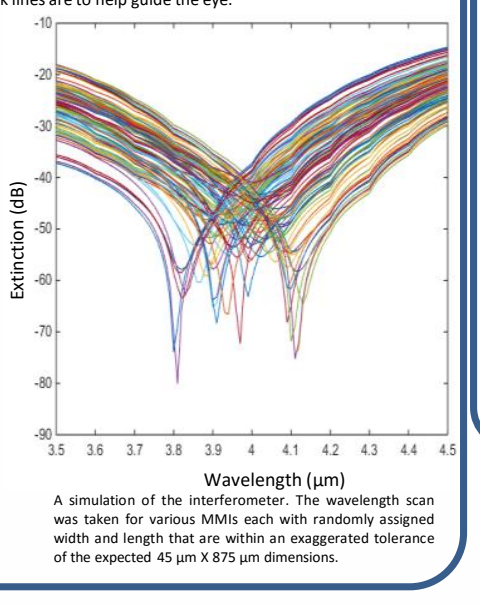

The Multimode interference coupler (MMI) is the key mechanism for the nulling interferometer. The beauty of the $\mathrm{MMI}$ is the simplicity of allowing light to interfere with itself in mathematically predictable patterns that reimage the light into identical images of the original wavefront. A special case $2 \times 2 \mathrm{MMI}$ is displayed, fabricated with tapering inputs from $2 \mu \mathrm{m}$ waveguides, maintaining a single mode, to $8 \mu \mathrm{m}$ increasing the fabrication tolerances and bandwidth of the device.

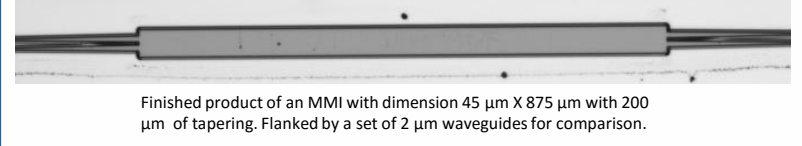

The interferometer is created in three layers of Chalcogenide glass (ChG) on a silica wafer. Initially starting with an under layer of $\mathrm{Ge}_{11.5} \mathrm{As}_{24} \mathrm{~S}_{64.5}$ and core layer of $\mathrm{Ge}_{11.5} \mathrm{As}_{24} \mathrm{Se}_{64.5}$ the device is plasma etched and then overclad with $\mathrm{Ge}_{11.5} \mathrm{As}_{24} \mathrm{~S}_{64.5}$. The light source used for the below tests was a custom built seeded optical parametric amplifier and a ND: $\mathrm{YVO}_{4}$ picosecond pump laser with a wavelength of $1064 \mathrm{~nm}$

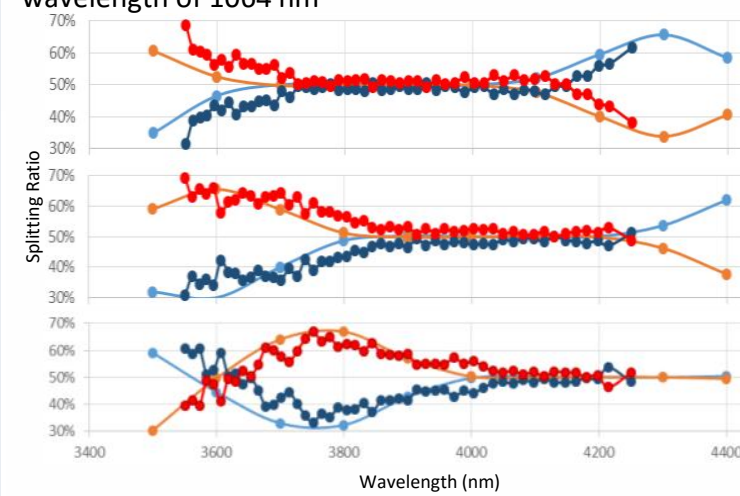

Measured results of the splitting ratio for MMI of various widths; $44 \mu \mathrm{m}, 45 \mu \mathrm{m}$ and $46 \mu \mathrm{m}$ respectively. The output ports are in red and dark blue, compared to the simulated results in orange and light blue.

R. N. Bracewell, "Detecting nonsolar planets by spinning infrared interferometer," Nature, 274, 780-781, 1978.

Madhusudhan, A. Burrows, and T. Currie, "Model Atmospheres for Massive Gas Giants With Thick Cloud Application To the Hr 8799 Planets and Predictions for Future Detections, Astrophys.J., 737, 34, 2011 Ma, D.-Y. Choi, Y. Yu, X. Gai, Z. Yang, S. Debbarma, S. Madden, and B. Luther-Davies, "Low-loss chalcogenide W. Soldano and E. C. M. Pennings, "Optical multi-mode interferes, 21, applications," J. Light. Technol., 13, 615-627, 1995 


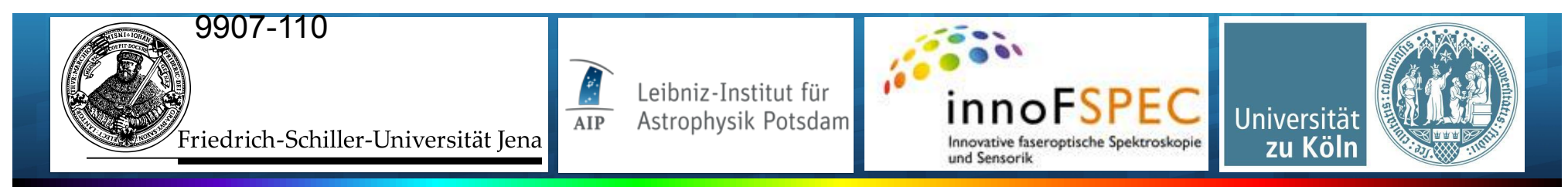

\title{
All-in-one 4-telescope beam combination with a zig-zag array of waveguides
}

\author{
Romina Diener ${ }^{1}$, Stefano Minardi ${ }^{1,2}$, Jan Tepper ${ }^{3}$, Stefan Nolte ${ }^{1}$, Lucas Labadie ${ }^{3}$ \\ Friedrich Schiller University Jena, Institute of Applied Physics, Albert Einstein Str. 15, 07745 Jena, Germany \\ 2 innoFSPEC, Leibnitz Institut für Astrophysik Potsdam, An der Sternwarte 16, 14482 Potsdam, Germany \\ University of Cologne, I. Physikalisches Institut, Zülpicherstr. 77, 50937 Cologne, Germany
}

Abstract

In this work we propose a new geometry of discrete beam combiners (DBC) for spectrally-resolved stellar interferometry which overcomes the limitations of previous designs. The new beam combiner is based on an array of coupled waveguides arranged in zig-zag pattern. It has been numerically optimized for the combination of 4 telescopes and engineered to operate in the L-band. We manufactured a first sample by direct laser writing in Gallium Lanthanum Sulfide glass, a highly transmissive material in the mid-infrared (550 nm to $10 \mu \mathrm{m}$ ). Initial near-field characterization of the fabricated sample at a wavelength of $3.4 \mu \mathrm{m}$ are encouraging, but highlighted the necessity of a better control of the polarization dispersion of individual waveguides, as well as induced stresses from manufacturing process.

\section{Zig-zag discrete beam combiners}

Discrete beam combiners (DBC) are 3D integrated optics multi-telescope, all-in-one interferometric beam combiners based on the properties of $2 \mathrm{D}$ arrays of evanescently coupled waveguides (photonic lattices) [1]. So far, only square or hexagonal waveguide arrays have been investigated both numerically and experimentally [2], which pose an obvious limitation as long as high-resolution spectro-interferometric applications are concerned [3]. Recent advance in the theory of DBCs [4], showed the necessity of diagonal (second order) coupling between waveguides in the $2 \mathrm{D}$ array in order to use the photonic lattice as a beam combiner. This discovery lead us to identify zig-zag photonic lattices (see Fig. 1) as ideal candidates enabling the interfacing of the DBC output to a spectrograph for high-resolution spectrointerferometry purposes.

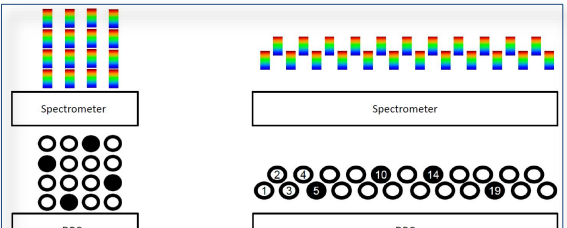

Fig. 1: (Left): Spectrometer output of $4 \times 4$ DBC. (Right): Spectrometer output of zig-zag DBC.

We optimized the design of the zig-zag array for 4-telescope combination, by numerical search of the DBC input configuration and array length which could deliver the most well conditioned Visibility to Pixel Matrix (V2PM) [5] for the beam combiner. It turned out that an array of 23 waveguides with the input configuration as shown in Fig. 1 and length $~ 1.4$ coupling lengths would result in the best beam combiner performance (condition number of V2PM = 3.6)

Manufacturing zig-zag DBC in Gallium Lanthanum Sulfide Our first sample of the zig-zag DBC was manufactured by ultrafast laser inscription (ULI) [6] in Gallium Lanthanum Sulfide (GLS), as we are targeting the realization of integrated optics beam combiners for mid-infrared [7]. With ULI, structural material modification is induced by intense and highly localized laser irradiation, which can be used to manufacture single-mode waveguides, as long as laser irradiation induces positive refractive index changes (like in GLS) $[7,8]$.

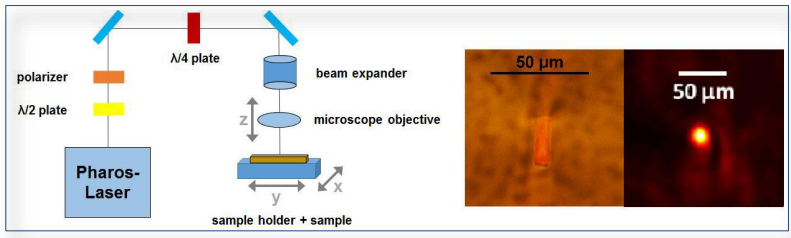

Fig. 2: (Left): Scheme of ULI setup. (Right): Image of a multi-pass waveguide \& guided mode at $\lambda=3.4 \mu \mathrm{m}$.

We manufactured waveguides supporting nearly circular mode fields at $\lambda=3.4 \mu \mathrm{m}$ by multipass ULI $[4,5]$ with the following writing parameters:

Laser source: Nd:KGW, 400 fs pulses Average power: $40 \mathrm{~mW}$

Repetition rate: $500 \mathrm{kHz}$

Translation velocity: $4 \mathrm{~mm} / \mathrm{s}$

Focusing objective NA: 0.35

Writing procedure: 21 parallel tracks with lateral separation of $300 \mathrm{~nm}$.

From the guided mode profile (Fig. 2), we estimated that the core-cladding refractive index contrast is in the range of $\Delta n \sim 10^{-3}$

To engineer the designed zig-zag DBC, we first estimated the coupling strength at $\lambda=3.4 \mu \mathrm{m}$ of pairs of evanescently coupled waveguides, by measuring the splitting ratios of a set of $2 \times 2$ couplers (Fig. 3) with waveguide gaps between 20 and $30 \mu \mathrm{m}$ and interaction length $\mathrm{L}=0,4$, and $8 \mathrm{~mm}$. Waveguides were both arranged in horizontal and diagonal geometry.

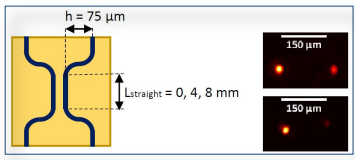

ig. 3. (Left). Schematic top view of the directional coupler. (Right): Image of the output intensities of a horizonta and diagonal directional coupler at $3.4 \mu \mathrm{m}$, separation ca. $23 \mu \mathrm{m}$, interaction length $4 \mathrm{~mm}$

According to the coupled mode theory, the splitting ratio of the coupled waveguide (cross)

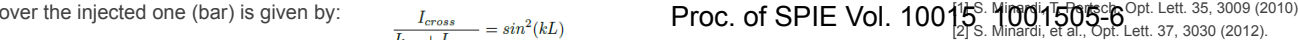

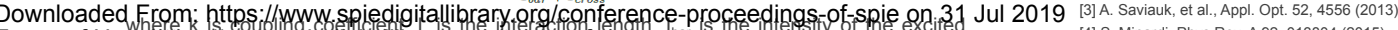
Terms of Us.

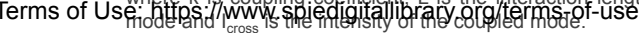

Estimates of the $\mathrm{kL}$ term from the measured splitting ratios have been plot as a function of the interaction length and fitted with a line to extract the the coupling strength as a function of the waveguide gap (see Fig. 4).

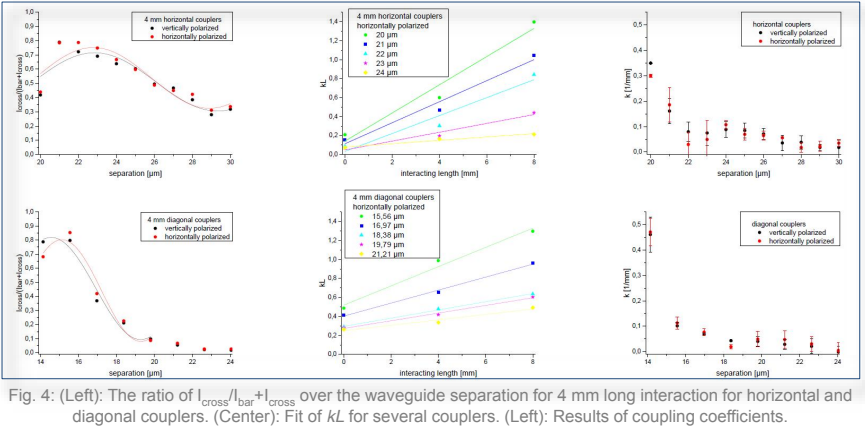

First results with zig-zag $D B C$

The measured coupling constants were used to engineer a sample of 4-T zig-zag DBC. We chose a horizontal period of $27 \mathrm{\mu m}$ to fulfill the coupled mode approximation for light propagation in the array. The overall sample length was $25 \mathrm{~mm}$. Output light distribution for input excitation of waveguides 5 and 19 of the manufactured component are shown in Fig. 5 for both vertically and horizontally polarized input laser beam. Comparison with numerical simulations showed however that manufacturing imperfections dominate light propagation in the array.

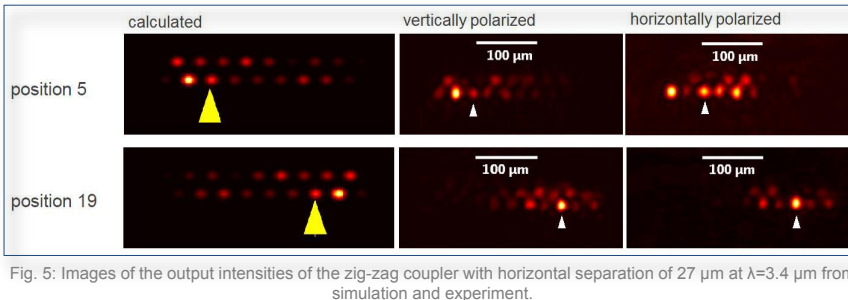

\section{Limitations of ULI}

Experimental data (Fig. 5) indicate an asymmetry and a strong polarization dependence of the output excitation patterns of the DBC. A possible explanation lies in the accumulation of mechanical stresses induced by ULI during the manufacturing process (the next waveguide is written on a substrate already modified by the manufacturing of previous ones). To verify this hypothesis, we used polarimetric strain measurements to gauge the impact of ULI-induced mechanical stresses. As shown in Fig. 6 , we found polarization rotation on a ca. 200-um-wide butterfly shaped area surrounding each written waveguide. Quantitative estimates of the optical path differences between orthogonal polarization states amount to $\sim 60 \mathrm{~nm}$ over 3-mmlong samples. This corresponds to a minimal stress-induced birefringence of $\Delta n \sim 2 \cdot 10^{-5}$ (assuming zero-order length).

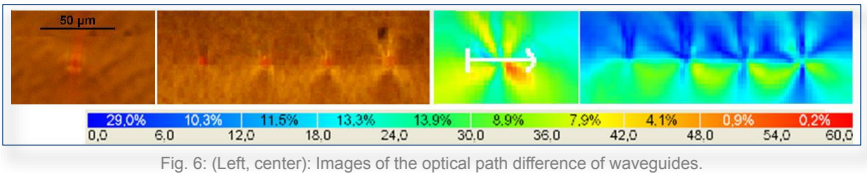

Conclusions and outlook

We have identified a new design of 4-T DBC, suitable for high-resolution spectrointerferometric applications (zig-zag DBC). However, manufactured samples of zig-zag DBC highlighted the need for a better control of ULI-induced mechanical stresses in the GLS substrate. We are currently investigating the possibility to cure stresses by a post-processing annealing [9]. Stress distribution by alternating the order of written waveguides is also being investigatec

E. Tatulli et al. Astronomy\&Astrophysics 464. 29 (2007)
R.R. Thomson, J. Allington-Smith Opt. Exp. (2009) M. Huges et al., Applied Physics Letters 90, 131113 (2007). 9] A. Arriola et al., Optics Express 21/3, pp. 2978-2986, 2013. 


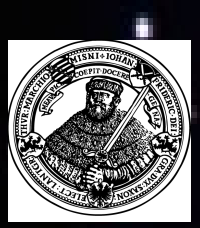

\section{6- and 8-Telescope Discrete Beam Combiners}

\section{Ronny Errmann ${ }^{a}$, Stefano Minardi ${ }^{a, b}$}

a Institute of Applied Physics, Abbe Center of Photonics, Friedrich Schiller University Jena, Max-Wien-Platz 1, 07743 Jena - Germany

${ }^{b}$ innoFSPEC, Leibnitz-Institut für Astrophysik Potsdam, An der Sternwarte 16, 14482 Potsdam - Germany

\section{Abstract}

We conduct an extensive numerical study to single out the best performing rectangular array of evanescently coupled waveguides (discrete beam combiner) that can be used as an integrated optic beam combiner for 6-telescopes at once. We found that the performance of a discrete beam combiner only depends on the conditioning of the Visibility to Pixel Matrix (V2PM) describing it. However, we found that the condition number of V2PM pertaining to different beam combiner architectures cannot be compared. We further report on the possible input waveguide configuration of an 8 -telescope discrete beam combiner featuring $8 \times 8$ or $9 \times 9$ waveguides.

Keywords: Integrated optics, coupled waveguides arrays, optical astrointerferometry

\section{Discrete Beam Combiners}

A Discrete Beam Combiner (DBC) is an array of evanescently coupled singlemode waveguides used to measure simultaneously the mutual coherence properties of light injected in selected sites [1]. Its application to astronomical interferometry could drastically reduce the complexity of multi-telescope integrated optics beam combiners [2]. For the efficient combination of the input fields $A_{k}$ from $N_{t}$ telescopes the DBC uses an array of $N_{w}>N_{t} \times N_{1}$ waveguides. Due to evanescent coupling, light propagating in one waveguide will leak to neighboring waveguides. At a given length of the array, the $\mathrm{N}_{\mathrm{t}}$ input fields will interfere inside the waveguides with variable amplitude and phase depending on the observed waveguide and the length of the array (see Fig. 1). The intensities of the $N_{w}$ waveguide modes at the end of the sample can be related to the complex visibilities $\Gamma_{i k}=\Gamma_{k i}{ }^{*}=\left\langle A A_{k}^{*}>\right.$ by a real valued, $N_{w} \times N_{t}$ elements matrix, the so called Visibility to Pixel Matrix (V2PM [3])

\section{$I_{n}=\sum_{i=1}^{N_{i}^{2}}(V 2 P M)_{n i} J_{i}$}

Here the complex visibilities $\Gamma$ enter the equation in the form of their quadratures $\mathrm{J}$ defined as:

$\vec{J}=\left|\Gamma_{1,1} \ldots \Gamma_{N, N} \Re \Gamma_{1,2} \ldots \Re \Gamma_{1, N} \ldots \Re \Gamma_{N-1, N} \Im \Gamma_{1,2} \ldots \Im \Gamma_{1, N} \ldots \Im \Gamma_{N-1, N}\right|^{T}$

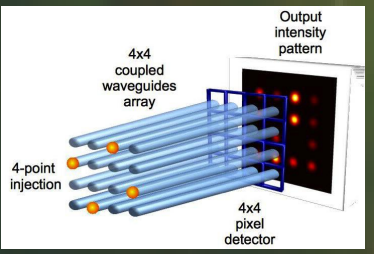

The quadratures $\mathrm{J}_{\mathrm{i}}$ can be retrieved by multiplying the I vector by the pseudoinverse of the V 2 PM (Pixel to Visibility Matrix, P2VM). The visibility modulus Matrix, P2VM). The visibility $n$ cal
can then be calculated from:

$$
V_{i j}=\sqrt{\frac{\left(\Re \Gamma_{i j}\right)^{2}+\left(\Im \Gamma_{i j}\right)^{2}}{\Gamma_{i i} \Gamma_{j j}}}
$$

Figure 1 - Cartoon illustrating the structure and basic setup of a 4 T-DBC.

\section{Goals and methods}

Aim of our investigation is a numerical optimization of the discrete beam combiner scheme suitable for the combination of $\mathbf{N}=6$ or $\mathbf{N}=8$ telescopes.

The chosen optimization parameters are the number of waveguides in the array and the relative strength of the diagonal coupling in the array. This choice is motivated by two previous results related to the DBC architecture, namely:

- Arrays featuring more than $\mathrm{N}_{t} \times \mathrm{N}_{t}$ waveguides have a better conditioned V2PM [4]. - Besides the horizontal coupling $C_{h}$, the diagonal coupling $C_{d}$ is a necessary condition for phase retrieval with arrays of waveguides [5]

As quality parameter for the optimal beam combination configuration, we chose the condition number (CN) of the V2PM. We verified (see Results 6T-DBC) that the conditioning of the V2PM is proportional to the error on the of the retrieved visibility amplitude in the photon-rich detection limit (inverse of the signal to noise ratio).

Two algorithms have been used in this work: a) a best DBC configuration finder, and b) a Monte-Carlo simulator of the visibility measurement procedure Block diagrams of the algorithms are shown here below

Algorithm (a)
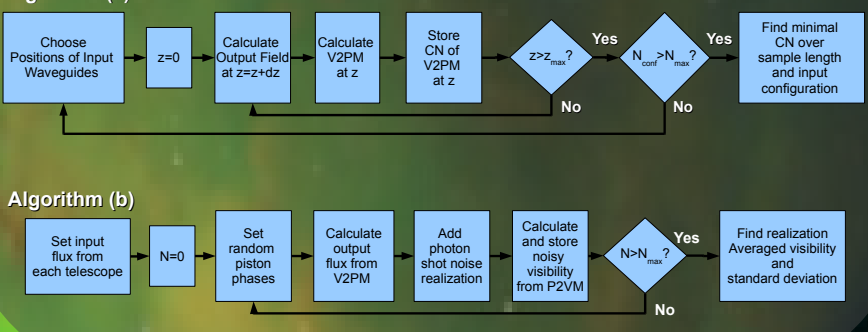

3. Results 6-T DBC
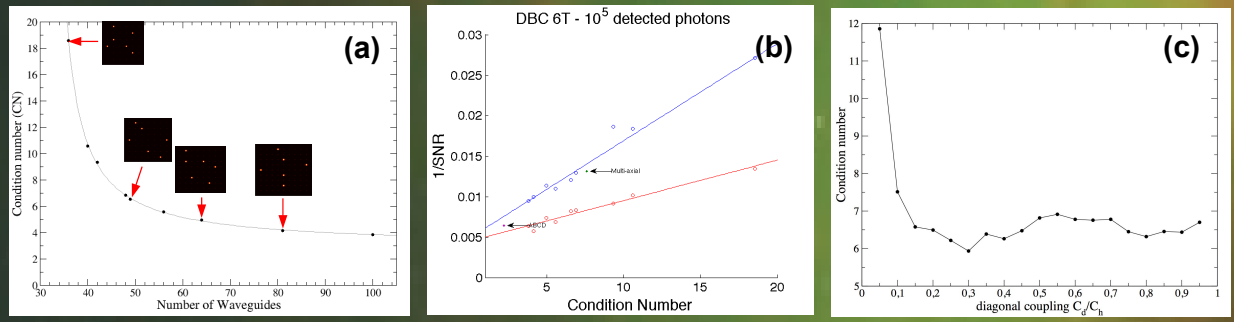

Figure 2 - We numerically investigated rectangular arrays of waveguides with increasing size to verify the existence of an array featuring a minimal $C N$ of the V2PM $\left(C_{d} / C_{h}=0.15\right)$. In the investigated size interval (from 36 to 100 waveguides) we found a monotonic decrease of the CN (Fig. 2.a). We verified that the measurement error on the visibility (unresolved source) is proportional only to the CN of the V2PM (Fig. 2.b). We found that different types of beam combiners (ABCD and multi-axial) have different dependencies of the measurement error on the CN of their V2PM (Fig. 2.b). For a given array (7x7) we tested the effect of the diagonal coupling on the conditioning of the 6T-DBC V2PM (Fig. 2.c). For $C / C>0.1$, diagonal coupling optimization has a relatively small impact on the conditioning of the V2PM $(<15 \%)$

\section{Conclusions and outlook}

- The $\mathrm{CN}$ of the V2PM is proportional to the error on the visibility amplitude retrieved from measurements.

- For DBCs larger arrays lead to smaller CN. For 6T-DBCs we could not find a minimum.

- The CN of the V2PM of different beam combiner architectures cannot be compared directly

Tuning of the ratio $C_{d} / C_{n}$ above $\sim 0.1$ can improve by $\sim 10-20 \%$ the conditioning of the V2PM of the DBC.

- Scaling of DBC beyond $8 \mathrm{~T}$ is limited by the factorial growth of the configurations to be tested.

We are currently investigating the performance of a new DBC geometry based on zig-zag arrays [6] and plan to manufacture and test first samples of 6T-DBC to verify numerical predictions.

\section{Acknowledgments}

4. Results 8-T DBC

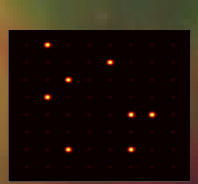

$8 \times 8$ array

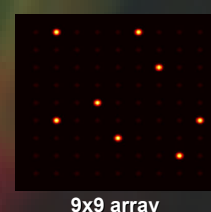

$9 \times 9$ array
Figure 3- Because of computational power limitations, we could only search for the $8 \mathrm{~T}$ input configurations in $8 \times 8$ and $9 \times 9$ arrays. Despite the high CN of the V2PM (see Table Despite the high CN of the V2PM (see Table error is comparable to that of the best $6 \mathrm{~T}$ DBC (see Fig. 2b).

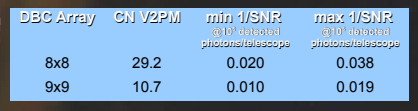

\section{References}

[1] Minardi, Pertsch Opt. Lett. 35, 3009 (2010).

[2] Benisty et al. A\&A 498, 601 (2009).

[3] Tatulli et al. A\&A 464, 29 (2007)

[4] Minardi MNRAS 422, 2656 (2012)

[5] Minardi Phys. Rev. A 92, 013804 (2015).

[6] Diener at al. SPIE 9907, poster 9907-110 (2016). 


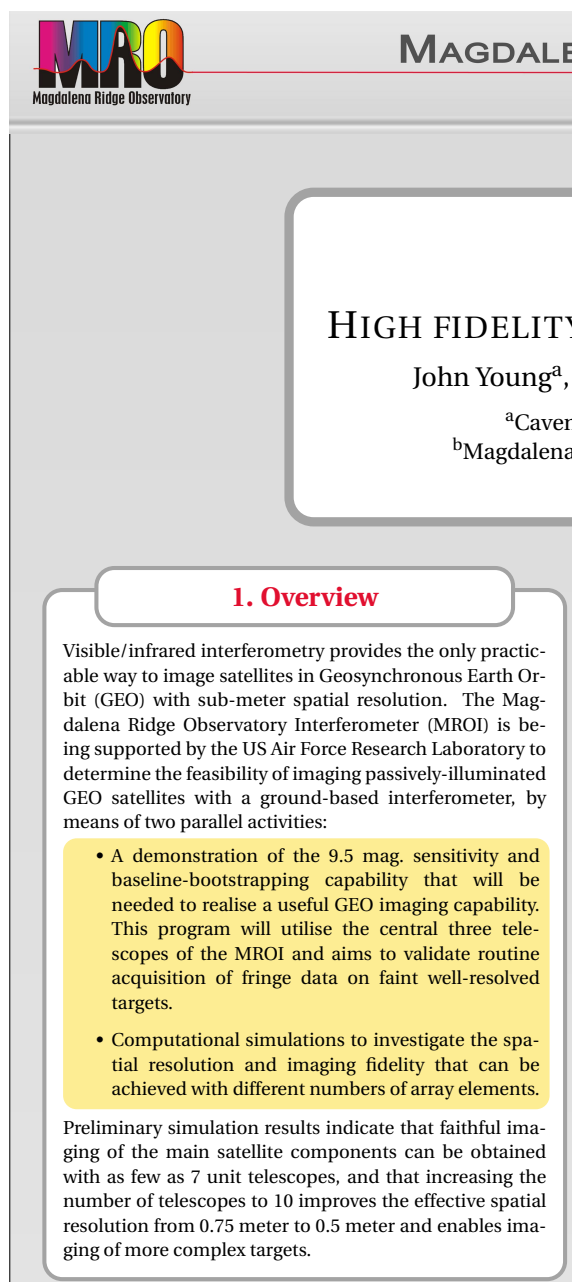

\section{Sensitivity Demonstration}

We have begun a five-year program to mitigate the principal technical risks associated with developing a groundbased satellite imaging array. Under this program, three unit telescopes will be installed on the Magdalena Ridge, unit telescopes will be installed on the Magdalena Ridge,
and used to secure interferometric data on GEO targets. These measurements will be used to validate models of the instrumental performance needed to predict the imaging capability of the full telescope array.

The MROI sub-systems will be deployed and tested in sequence, starting with the unit telescopes, beam relay system, and delay lines. The program will culminate in measurements of co-phased visibilities (year four) and closure phases (year five) for GEO targets.

MROI has been designed to accommodate up to $10 \mathrm{unit}$ telescopes. For the simulations presented here, we have also considered a 19-telescope array - this would require changes to the existing designs and site infrastructure.

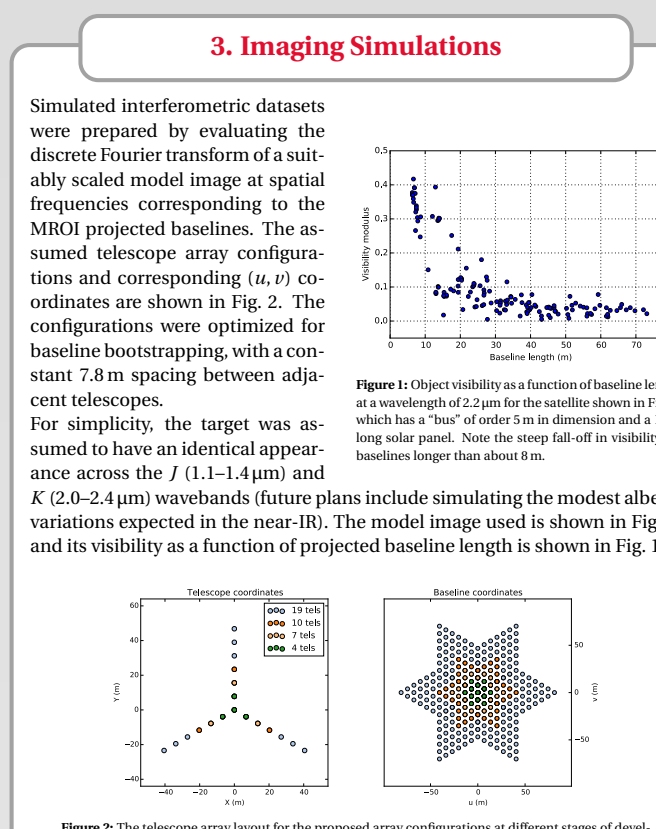

Figure 2: The telescope array layout for the proposed array configurations at different stages of devel-
opment (left) and the $(u, v)$ plane coverage of these arrays (right). Only the most compact configuraopment (left) and the $(u, v)$ plane

The Fourier data were then used to generate interferometric observables (squared visibilities and bispectra). Random errors were added to the data based on realistic assumptions of co-phasing accuracy at the assumed 9.5 mag. target brightness. In addition, uncorrelated calibration errors were added to the visibilities $\left(\Delta V^{2} / V^{2}=0.02\right)$ and closure phases $\left(0.8^{\circ}\right)$. For the tentelescope simulation, the mean SNR of the synthetic $V^{2}$ was 6.8 , and the mean closure phase error was $3.1^{\circ}$. The largest closure phase errors were $\sim 40^{\circ}$.

The simulated data were used as input to the BSMEM image reconstruction The simulated data were used as input to the BSMEM image reconstruction
code. ${ }^{1}$ For each dataset, a two-step reconstruction procedure was used. First code. $^{1}$ For each dataset, a two-step reconstruction procedure was used. First,
higher spatial frequency data were removed and BSMEM run with an uninformative circular Gaussian prior image. High spatial frequencies and noise were removed from the BSMEM output image by convolving with a circular Gaussian function and setting pixel values below a user-selected threshold to zero. The resulting image was used as a prior for a second run of BSMEM on the full dataset.

\section{Simulation Results}

The model spacecraft has structure on a range of scales from $\sim 0.2 \mathrm{~m}$ to $17 \mathrm{~m}$ and is therefore a good test of the accuracy and effective resolution with which images can be reconstructed. For arrays of 7 or more telescopes, the simulation results (Fig. 3) show faithful reconstructions. The effective spatial resolution of the images increases as the number of telescopes, and hence the maximum baseline, is increased. These images exhibit "superresolution" beyond the nominal $\lambda / B$ diffraction limit. For example, the $1 \mathrm{~m}$ diameter structure at the tip of the boom is reample, the $1 \mathrm{~m}$ diameter structure at the tip of the boom is re-
solved in the seven-telescope image where the nominal resolution is just $1.6 \mathrm{~m}$. Observations using fewer than 7 telescopes provide information on the size and orientation of the satellite but do not permit model-independent imaging.
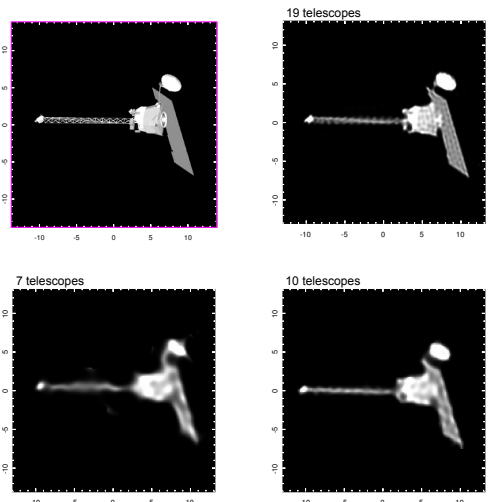

Figure 3: Truth (top left) and reconstructed images using 19, 10 , and 7 telescope
(clockwise from top right) for a simulated MROI observation of a GEO satellite. The axes are scaled in meter

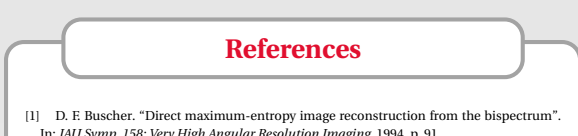

In: IAU Symp. 158: Very High Angular Resolution Imaging. 1994, p. 91 . 


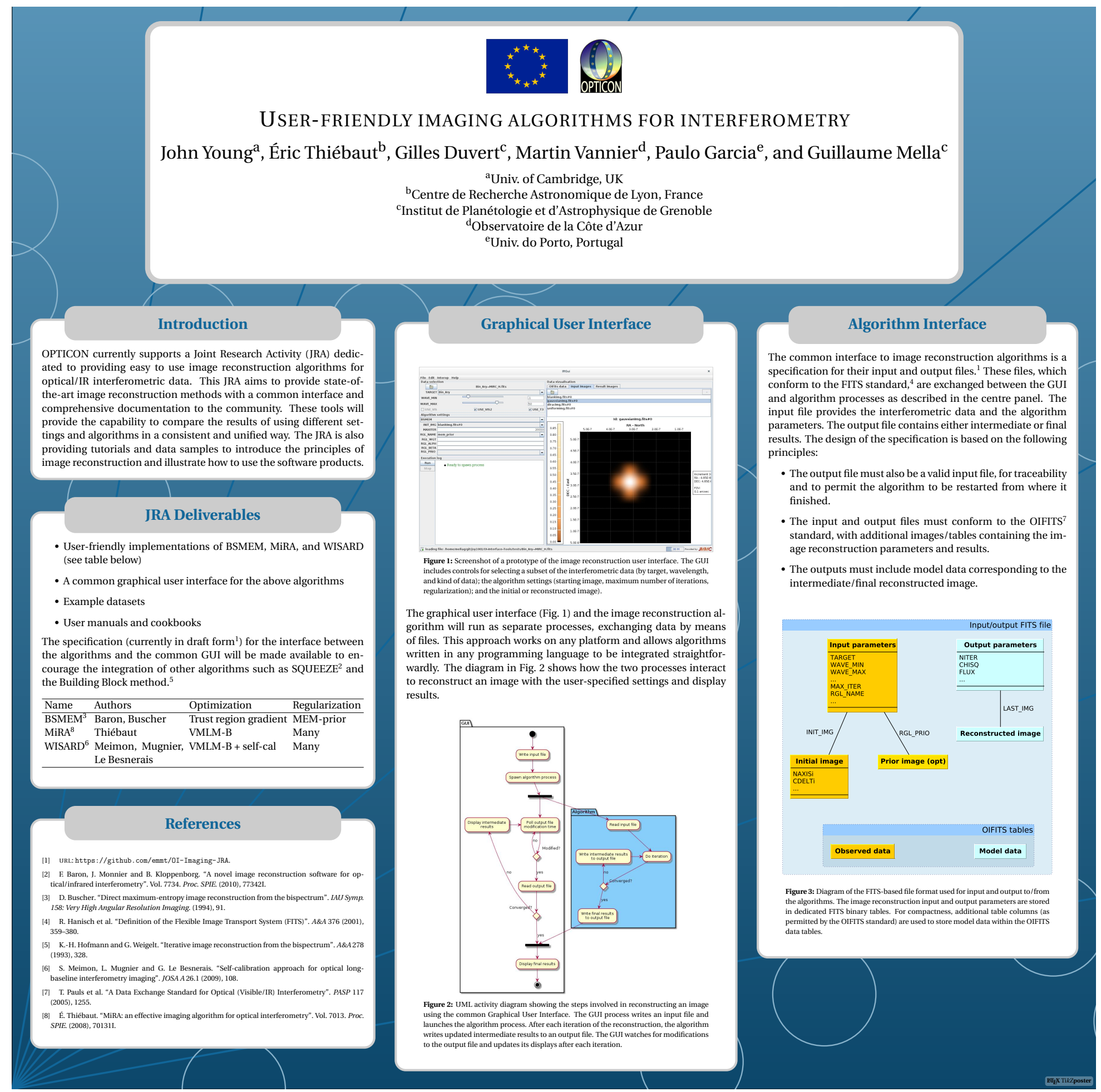




\section{AGP (Astrometric Gravitation Probe) Optical Design Report}

Alberto Riva, Mario Gai, Federico Landini, Paolo Lazzarini, Daniele Gallieni, Matteo Tintori, Alberto Anselmi, Stefano Cesare, Deborah Busonero, Mario Gilberto Lattanzi, Alberto Vecchiato

9907-150

This paper describes the current opto-mechanical design of AGP, a mission designed for astrometric verification of General Relativity (GR) and competing gravitation theories by means of precise determination of light de- flection on field stars, and of orbital parameters of selected Solar System objects. The optical concept includes a planar rear-view mirror for simultaneous imaging on the CCD mosaic detector of fields of view also from the direction opposite to the Sun, affected by negligible deflection, for the sake of real time calibration. The precision of astrometric measurements on individual stars will be of order of 1 mas, over two fields separated by few degrees around the Sun and observed simultaneously. We describe the optical design characteristics, with particular reference to manufacturing and tolerancing aspects, evidencing the preservation of very good imaging performance over the range of expected operating conditions.

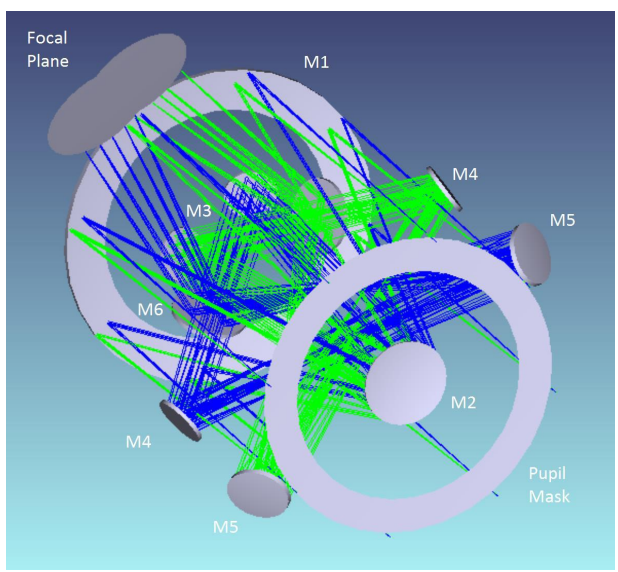

The implementation of the AGP differential measurement scheme is achieved by means of a dualline-of-sight telescope with a diluted pupil mask, used in order to reject the Sun light. Rear-view mirror picks-up the star light of sources opposite to the sun, and uses the non populated part of the mirrors in order to drive the light into the "front" optical path.

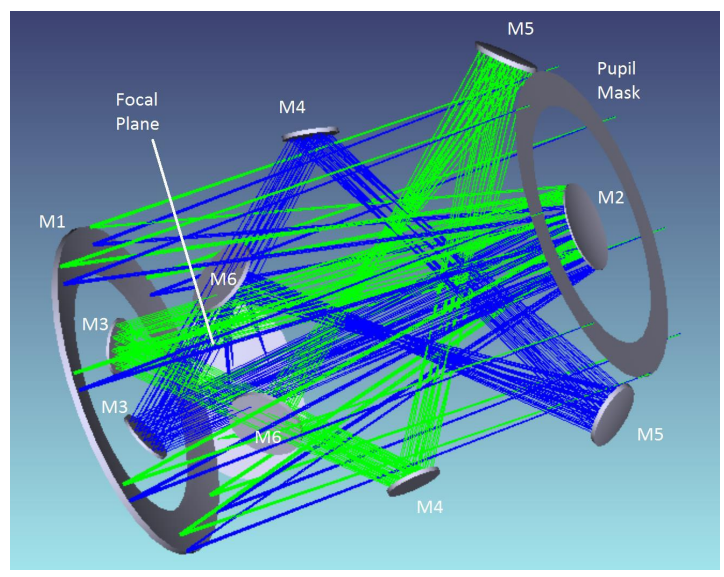

The AGP telescope is designed with two channels, but it is compatible with up to four/eight channels. The two channels are pointing symmetrically to about +/- 1 deg with respect to the optical axis of the telescope, which in nominal operation is set on the Sun. This corresponds to a base angle of 2 deg. Each channel has a field of view of $16^{\prime} \times 16^{\prime}$. Each channel has a similar and symmetric layout with respect to the telescope incoming beam.

The AGP structure is composed by the following elements:

- Two circular extremities built from titanium

- A large Carbon Fiber Reinforced Plastics (CFRP) central element.

- A CFRP removable.

- Two CFRP open boxes, obtained by the same mold. These two elements provide the housing for the two M4s and M5s

- The four legs, built from stainless steel welded pipes. The extremities of three of the four legs end with flexible elements, to separate the thermal deformations of the AGP module from those of the satellite.
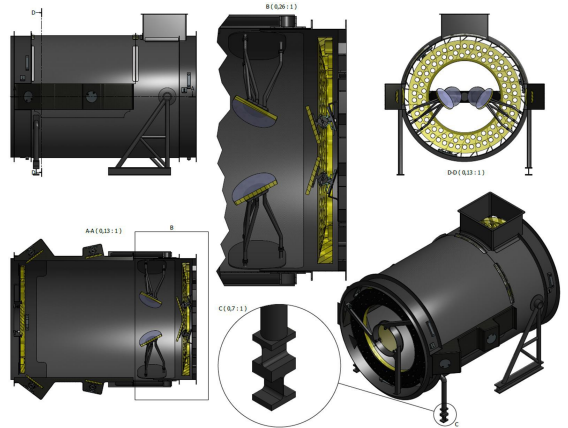

A first study of the structure has been performed by FEA. In the model the CFRP parts are modeled using an equivalent isotropic material. The interfaces to the mirror have a good level of detail and a first computation of the stress, even if still purely indicative, is thus realistic.

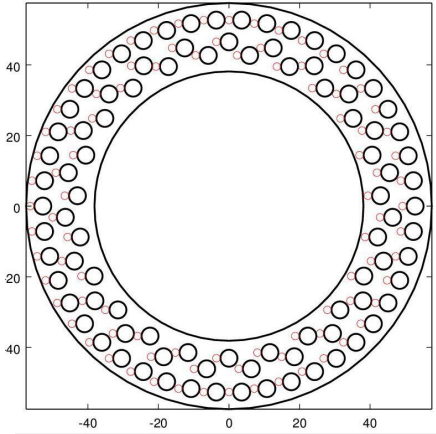

Primary mirror holes-set with footprint of pupil mask

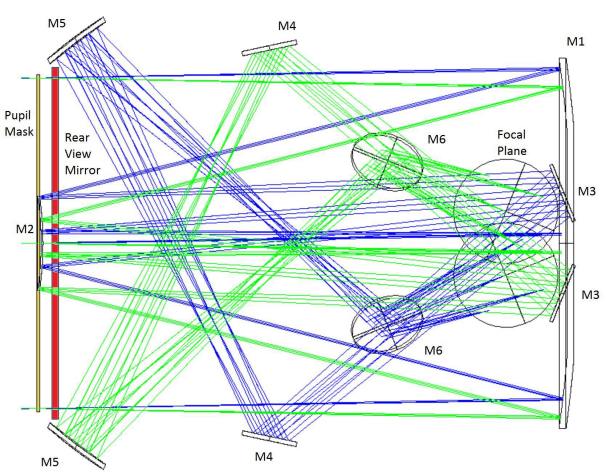

Rear-view mirror displacement
Rear-view mirror: according to AGP measurements principles, star light coming from sources in the direction opposite to the Sun, are not affected by any deflection, and should improve the calibration issues of the experiment. The focal plane therefore images at the same time the two front and the two rear fields of view.

CONCLUSIONS: The present paper summarizes the Optical Design for the AGP mission, whose main goal is the measure of positions of stars for light deffeøtione 